\title{
Acyclic sets in $k$-majority tournaments
}

\author{
Kevin G. Milans*, Daniel H. Schreiber ${ }^{\dagger}$, Douglas B. West $t^{\ddagger}$ \\ Submitted: Jul 31, 2010; Accepted: May 18, 2011; Published: May 30, 2011 \\ Mathematics Subject Classification: 05C20, 06A05
}

\begin{abstract}
When $\Pi$ is a set of $k$ linear orders on a ground set $X$, and $k$ is odd, the $k$ majority tournament generated by $\Pi$ has vertex set $X$ and has an edge from $u$ to $v$ if and only if a majority of the orders in $\Pi$ rank $u$ before $v$. Let $f_{k}(n)$ be the minimum, over all $k$-majority tournaments with $n$ vertices, of the maximum order of an induced transitive subtournament. We prove that $f_{3}(n) \geq \sqrt{n}$ always and that $f_{3}(n) \leq 2 \sqrt{n}-1$ when $n$ is a perfect square. We also prove that $f_{5}(n) \geq n^{1 / 4}$. For general $k$, we prove that $n^{c_{k}} \leq f_{k}(n) \leq n^{d_{k}(n)}$, where $c_{k}=3^{-(k-1) / 2}$ and $d_{k}(n) \rightarrow \frac{1+\lg \lg k}{-1+\lg k}$ as $n \rightarrow \infty$.
\end{abstract}

\section{Introduction}

When $\Pi$ is a set of linear orders on a ground set $X$, the majority digraph of $\Pi$ has vertex set $X$ and has an edge from $u$ to $v$ if and only if a majority of the orders in $\Pi$ rank $u$ before $v$. When $\Pi$ has size $k$ and $k$ is odd, the majority digraph is a $k$-majority tournament. A $k$-majority tournament is a model of the consensus preferences of a group of $k$ individuals.

In studying generalized voting paradoxes, McGarvey [8] showed that every $n$-vertex tournament is realizable as a $k$-majority tournament with $k=2\left(\begin{array}{l}n \\ 2\end{array}\right)$. Erdös and Moser [6] improved this by showing that $k=O(n / \log n)$ always suffices, and Stearns [9] showed that $k=\Omega(n / \log n)$ is sometimes necessary.

In addition to modeling group preferences using a small number of criteria, the $k$ majority tournaments for fixed $k$ form a well-behaved class of tournaments. For example, consider domination. The domination number of a directed graph $D$, denoted $\gamma(D)$, is the minimum size of a vertex subset $S$ such that each vertex not in $S$ has an immediate

*Department of Mathematics, University of South Carolina, Columbia, SC 29208, milans@math.uiuc.edu. Research supported by the National Science Foundation grant DMS 08-38434 "EMSW21-MCTP: Research Experience for Graduate Students".

$\dagger$ Daniel Schreiber tragically passed away on July 27, 2010.

$\ddagger$ Department of Mathematics, University of Illinois, Urbana, IL 61801, west@math.uiuc.edu. Research supported by National Security Agency Award No. H98230-10-1-0363. 
predecessor in $S$. In general, Erdős [5] showed that $n$-vertex tournaments can have domination number $\Omega(\log n)$. In contrast, for $k$-majority tournaments the domination number is bounded; Alon et al. [1] proved that every $k$-majority tournament has domination number at most $O(k \log k)$ and constructed $k$-majority tournaments with domination number at least $\Omega(k / \log k)$.

A set of vertices in a tournament is acyclic if the subtournament induced by it contains no cycle. Let $a(D)$ denote the maximum size of an acyclic set in $D$. Erdös and Moser [6] showed that every $n$-vertex tournament has an acyclic set of size at least $\lfloor\lg n\rfloor+1$, where "lg" denotes $\log _{2}$. Furthermore, they showed that almost every $n$-vertex tournament $T$ satisfies $a(T) \leq 2\lfloor\lg n\rfloor+1$.

In contrast, every $n$-vertex $k$-majority tournament has an acyclic set whose size is bounded below by a polynomial in $n$. Let

$$
f_{k}(n)=\min \{a(T): T \text { is an } n \text {-vertex } k \text {-majority tournament }\} .
$$

We prove that $f_{3}(n) \geq \sqrt{n}$ always and that $f_{3}(n) \leq 2 \sqrt{n}-1$ when $n$ is a perfect square. We also prove that $f_{5}(n) \geq n^{1 / 4}$. For general $k$, we prove that $n^{c_{k}} \leq f_{k}(n) \leq n^{d_{k}}$, where $c_{k}=3^{-(k-1) / 2}$ and $d_{k}(n) \rightarrow \frac{1+\lg \lg k}{-1+\lg k}$ as $n \rightarrow \infty$. In proving the upper bound on $f_{k}(n)$, we use the existence of an $r$-vertex tournament $T$ with $a(T) \leq 2 \lg r+1$.

In discussing acyclic sets in tournaments, we use the elementary characterizations of such sets. A set is acyclic if and only if the subtournament induced by it is transitive, which holds if and only if it induces no triangle, where a triangle is a (directed) 3-cycle. We also use the Erdős-Szekeres Theorem.

Theorem (Erdös-Szekeres [7]). Every list of more than $(r-1)(s-1)$ distinct integers has an increasing sublist of length $r$ or a decreasing sublist of length $s$.

Let $\Pi$ be a set of linear orderings of a ground set $X$. A set of elements of $X$ is $\Pi$ consistent if it appears in the same order in each member of $\Pi$. When $\Pi$ has even size, a set $S$ of elements of $X$ is $\Pi$-neutral if for all distinct $u, v \in S$, element $u$ appears before element $v$ in exactly half the members of $\Pi$. Note that if $S$ is $\left\{\pi_{1}, \pi_{2}\right\}$-neutral, then $\pi_{1}$ ranks the elements of $S$ in reverse order from $\pi_{2}$. We use the following rephrasing of the Erdős-Szekeres Theorem.

Theorem (Erdös-Szekeres [7]). Given linear orderings $\pi_{1}$ and $\pi_{2}$ of a set $X$ with $|X|>$ $(r-1)(s-1)$, there is a $\left\{\pi_{1}, \pi_{2}\right\}$-consistent set of size $r$ or a $\left\{\pi_{1}, \pi_{2}\right\}$-neutral set of size $s$.

Proof. Rename the elements of $X$ so that $\pi_{1}$ is the identity ordering $(1, \ldots, n)$, and apply the Erdős-Szekeres Theorem to $\pi_{2}$.

Acyclic sets in tournaments are related to independent sets and cliques in graphs; let $\alpha(G)$ and $\omega(G)$ denote the maximum sizes of a clique and an independence set in a graph $G$, respectively. Let $[n]=\{1, \ldots, n\}$. Graphs and tournaments with vertex set $[n]$ correspond as follows: two vertices are adjacent in $G$ if and only if the edge joining them in $T$ points from the smaller vertex to the larger. Every clique or independent set in $G$ is acyclic in $T$, so $a(T) \geq \max \{\alpha(G), \omega(G)\}$. 
Although acyclic sets in $T$ need not be cliques or independent sets in $G$, still the Erdős-Szekeres Theorem yields an upper bound. Let $S$ be a largest acyclic set in $T$. Let $\pi_{1}$ be the restriction to $S$ of the usual ordering of $[n]$, and let $\pi_{2}$ be the transitive order formed by $S$ in $T$. Now any $\left\{\pi_{1}, \pi_{2}\right\}$-neutral set is an independent set in $G$, and any $\left\{\pi_{1}, \pi_{2}\right\}$-consistent set is a clique in $G$. Hence the Erdös-Szekeres Theorem implies $\max \{\alpha(G), \omega(G)\} \geq \sqrt{|S|}$, or $a(T) \leq(\max \{\alpha(G), \omega(G)\})^{2}$.

\section{$2 \quad k=3$ and $k=5$}

In this section, we prove bounds on $f_{k}(n)$ when $k$ is 3 or 5 . When $k=3$, our upper and lower bounds differ only by a factor of 2 .

Beame and Huynh-Ngoc [3] gave a simple argument that when $\left\{\pi_{1}, \pi_{2}, \pi_{3}\right\}$ is a set of three orderings of $[n]$, there is a $\left\{\pi_{i}, \pi_{j}\right\}$-consistent set of size $n^{1 / 3}$ for some $i, j \in\{1,2,3\}$. Beame, Blais, and Huynh-Ngoc [2] proved that for integers $n$ and $k$ with $k \geq 3$ and $n \geq k^{2}$, there is a set of $k$ orderings of $[n]$ in which no two orderings have a consistent set of size greater than $16(n k)^{1 / 3}$.

When two of three orderings are consistent on a set, that set is acyclic in the resulting 3-majority tournament. Thus $f_{3}(n) \geq n^{1 / 3}$ using only sets that are consistent in two of the orders. By considering also acyclic sets that are neutral in the first two orders, we improve the lower bound.

Proposition 2.1. $f_{3}(n) \geq \sqrt{n}$.

Proof. Let $T$ be an $n$-vertex 3-majority tournament realized by $\left\{\pi_{1}, \pi_{2}, \pi_{3}\right\}$. By the ErdösSzekeres Theorem, there is a $\left\{\pi_{1}, \pi_{2}\right\}$-consistent set of size at least $\sqrt{n}$ or a $\left\{\pi_{1}, \pi_{2}\right\}$-neutral set of size at least $\sqrt{n}$. In the first case, this set is acyclic.

Otherwise, let $S$ be a $\left\{\pi_{1}, \pi_{2}\right\}$-neutral set of size at least $\sqrt{n}$. Since $S$ is $\left\{\pi_{1}, \pi_{2}\right\}$ neutral, it follows that $S$ induces a transitive subtournament of $T$ with vertices in the same order as in $\pi_{3}$. Hence $S$ is acyclic.

Despite the simplicity of Proposition 2.1, the bound is not far from optimal.

Theorem 2.2. If $n$ is a perfect square, then $f_{3}(n) \leq 2 \sqrt{n}-1$.

Proof. Let $n=r^{2}$, and let $X=[r] \times[r]$. View $X$ as points in the first quadrant of the plane, so that $\left(x_{1}, x_{2}\right)$ gives (column, row) index pairs. We define orderings $\pi_{1}, \pi_{2}, \pi_{3}$ of $X$ and argue that $a(T) \leq 2 r-1$, where $T$ is the resulting 3-majority tournament on $X$.

$$
\begin{aligned}
& \left(u_{1}, u_{2}\right)<\left(v_{1}, v_{2}\right) \text { in } \pi_{1} \Longleftrightarrow u_{2}<v_{2} \text { or }\left(u_{2}=v_{2} \text { and } u_{1}<v_{1}\right) \\
& \left(u_{1}, u_{2}\right)<\left(v_{1}, v_{2}\right) \text { in } \pi_{2} \Longleftrightarrow u_{2}>v_{2} \text { or }\left(u_{2}=v_{2} \text { and } u_{1}<v_{1}\right) \\
& \left(u_{1}, u_{2}\right)<\left(v_{1}, v_{2}\right) \text { in } \pi_{3} \Longleftrightarrow u_{1}>v_{1} \text { or }\left(u_{1}=v_{1} \text { and } u_{2}<v_{2}\right) .
\end{aligned}
$$

Since these are all lexicographic orderings (up to symmetry), they are linear orderings.

Consider distinct vertices $u$ and $v$, with $u=\left(u_{1}, u_{2}\right)$ and $v=\left(v_{1}, v_{2}\right)$. If $u$ and $v$ differ in both coordinates, then $u v \in E(T)$ if and only if $u_{1}>v_{1}$. Indeed, $\{u, v\}$ is 
$\left\{\pi_{1}, \pi_{2}\right\}$-neutral; $\pi_{3}$ breaks the tie by putting the vertex with larger first coordinate first. If $u_{2}=v_{2}$, then $u v \in E(T)$ if and only if $u_{1}<v_{1}$. If $u_{1}=v_{1}$, then $u v \in E(T)$ if and only if $u_{2}<v_{2}$.

For $i, j \in[r]$, let $R_{i}=\left\{\left(u_{1}, u_{2}\right) \in X: u_{2}=i\right\}$ and $C_{j}=\left\{\left(u_{1}, u_{2}\right) \in X: u_{1}=j\right\}$. Let $S$ be an acyclic subset of $T$. We prove $|S| \leq 2 r-1$ by mapping the vertices in $S$ to represent distinct elements of $\left\{R_{i}: i \in[r]-\{1\}\right\} \cup\left\{C_{j}: j \in[r]\right\}$. For each column $C_{j}$ that intersects $S$, let the lowest vertex in $S \cap C_{j}$ (smallest second coordinate) represent $C_{j}$. Every other vertex in $S$ represents the row containing it. No vertex represents $R_{1}$, because this vertex would be the lowest in its column and represent the column instead.

By construction, no two vertices represent the same column. If two vertices $u$ and $v$ represent the same row $R_{i}$, then $u=\left(u_{1}, i\right)$ and $v=\left(v_{1}, i\right)$; we may assume that $u_{1}<v_{1}$. Since $u$ represents $R_{i}$, some vertex $w$ in $S$ is in the same column as $u$ but has a smaller second coordinate. That is, $w=\left(u_{1}, k\right)$ with $k<i$. Now $u v, v w$, and $w u$ are edges in $T$, contradicting that $S$ is an acyclic set.

Proposition 2.1 and Theorem 2.2 combine to give general bounds on $f_{3}(n)$.

Corollary 2.3. $\sqrt{n} \leq f_{3}(n)<2 \sqrt{n}+1$.

Proof. The lower bound is Proposition 2.1. For the upper bound, let $n^{\prime}$ be the smallest perfect square that is at least $n$; note that $\sqrt{n^{\prime}}-\sqrt{n}<1$. By the monotonicity of $f$ and Theorem 2.2, $f_{3}(n) \leq f_{3}\left(n^{\prime}\right) \leq 2 \sqrt{n^{\prime}}-1<2 \sqrt{n}+1$.

We now consider $k=5$. Because adding a linear ordering and its reverse to $\Pi$ does not change the majority digraph, every $k$-majority tournament is a $(k+2)$-majority tournament, and hence $f_{k+2}(n) \leq f_{k}(n)$. This observation yields the best upper bound we currently have on $f_{5}(n)$, which is $f_{5}(n) \leq f_{3}(n)<2 \sqrt{n}+1$. One would expect $f_{5}(n)$ to be strictly smaller than $f_{3}(n)$, and indeed our lower bound for $f_{5}(n)$ is smaller than that for $f_{3}(n)$. We use the well-known fact that any poset of size $r$ has a chain or an antichain of size at least $\sqrt{r}$ (by Dilworth's Theorem, for example [4]).

Theorem 2.4. $f_{5}(n) \geq n^{1 / 4}$.

Proof. Let $T$ be an $n$-vertex 5 -majority tournament realized by $\left\{\pi_{1}, \ldots, \pi_{5}\right\}$. Apply the Erdős-Szekeres Theorem to $\pi_{1}$ and $\pi_{2}$ to obtain a $\left\{\pi_{1}, \pi_{2}\right\}$-consistent or a $\left\{\pi_{1}, \pi_{2}\right\}$-neutral set $S$ of size at least $\sqrt{n}$. Let $r=|S|$. If $S$ is $\left\{\pi_{1}, \pi_{2}\right\}$-neutral, then the subtournament on $S$ is an $r$-vertex 3-majority tournament realized by $\left\{\pi_{3}, \pi_{4}, \pi_{5}\right\}$. By Proposition 2.1, $S$ contains an acyclic set of size $\sqrt{r}$, and therefore $a(T) \geq n^{1 / 4}$.

Otherwise, $S$ is $\left\{\pi_{1}, \pi_{2}\right\}$-consistent. Let $P$ be the poset that is the intersection of the orders $\pi_{3}, \pi_{4}$, and $\pi_{5}$, so $u<_{P} v$ if and only if all three orders list $u$ before $v$. Let $P^{\prime}$ be the subposet of $P$ on $S$. The elements of any chain of size at least $\sqrt{r}$ in $P^{\prime}$ form a $\left\{\pi_{3}, \pi_{4}, \pi_{5}\right\}$-consistent set, and this set is acyclic in $T$.

If there is no such chain, then $P^{\prime}$ has an antichain $A$ of size at least $\sqrt{r}$. Any two elements of $A$ appear in both orders among $\left\{\pi_{3}, \pi_{4}, \pi_{5}\right\}$. Thus, $A$ induces a transitive subtournament, ordered by the common restriction to $A$ of $\pi_{1}$ and $\pi_{2}$. Again $a(T) \geq n^{1 / 4}$. 


\section{General odd $k$}

In this section we present bounds on $f_{k}(n)$ for general $k$. Our bounds are far apart when $k$ is large, but they do show that $f_{k}(n)$ has polynomial growth (between powers of $n$ ) for all fixed $k$. The exponents on $n$ in the upper and lower bounds tend to zero as $k$ grows.

Given a family $\Pi$ of linear orders on $[n]$, a set $S \subseteq[n]$ is $\Pi$-homogeneous if there is a linear order $L$ on $S$ and an integer $h$ such that exactly $h$ members of $\Pi$ list $u$ before $v$ whenever $u<_{L} v$. Relative to $L$, we then say that $h$ is the signature of $S$. When $|\Pi|$ is odd, a $\Pi$-homogeneous set is acyclic in the resulting $|\Pi|$-majority tournament. Our argument for the lower bound finds a П-homogeneous set inductively.

Theorem 3.1. Let $k$ be an odd integer. For any family $\Pi$ of $k$ linear orders on an $n$-set, there is a П-homogeneous set of size at least $n^{c_{k}}$, where $c_{k}=3^{-(k-1) / 2}$; hence $f_{k}(n) \geq n^{c_{k}}$.

Proof. We use induction on $k$; the claim is trivial for $k=1$. For $k \geq 3$, let $\Pi=$ $\left\{\pi_{1}, \ldots, \pi_{k}\right\}$. By the Erdős-Szekeres Theorem, there is a $\left\{\pi_{k-1}, \pi_{k}\right\}$-consistent set of size at least $n^{2 / 3}$ or a $\left\{\pi_{k-1}, \pi_{k}\right\}$-neutral set of size at least $n^{1 / 3}$. Call this set $S$, and let $\Pi^{\prime}=\left\{\pi_{1}^{\prime}, \ldots, \pi_{k-2}^{\prime}\right\}$, where $\pi_{j}^{\prime}$ is the restriction of $\pi_{j}$ to $S$. The induction hypothesis yields within $S$ a $\Pi^{\prime}$-homogeneous set $S^{\prime}$ of size at least $|S|^{c_{k-2}}$.

If $S$ is $\left\{\pi_{k-1}, \pi_{k}\right\}$-neutral, then $S^{\prime}$ is not only $\Pi^{\prime}$-homogeneous but also $\Pi$-homogeneous. We have $\left|S^{\prime}\right| \geq n^{c_{k-2} / 3}$, which suffices since $c_{k}=c_{k-2} / 3$.

Hence we may assume that $S$ is $\left\{\pi_{k-1}, \pi_{k}\right\}$-consistent. We cannot conclude that $S^{\prime}$ is $\Pi$-homogeneous, because the ordering $L_{1}$ under which $S^{\prime}$ is $\Pi^{\prime}$-homogeneous may differ from the common ordering $L_{2}$ of $S^{\prime}$ in $\pi_{k-1}$ and $\pi_{k}$. Applying the Erdös-Szekeres Theorem to $L_{1}$ and $L_{2}$ yields an $\left\{L_{1}, L_{2}\right\}$-consistent or $\left\{L_{1}, L_{2}\right\}$-neutral set $S^{\prime \prime}$ of size at least $\sqrt{\left|S^{\prime}\right|}$.

Let $h$ be the signature of $S^{\prime}$ relative to $L_{1}$. Whether $S^{\prime \prime}$ is $\left\{L_{1}, L_{2}\right\}$-consistent or $\left\{L_{1}, L_{2}\right\}$-neutral, $S^{\prime \prime}$ is $\Pi$-homogeneous relative to $L_{1}$ with signature $h+2$ or $h-2$, respectively. Furthermore, $\left|S^{\prime \prime}\right| \geq \sqrt{\left|S^{\prime}\right|} \geq\left(\left(n^{2 / 3}\right)^{c_{k-2}}\right)^{1 / 2} \geq n^{c_{k-2} / 3}=n^{c_{k}}$.

Our upper bound on $f_{k}(n)$ for general odd $k$ uses induction on $n$. We begin with a $(k+1) / 2$-vertex tournament $T_{1}$ having no large acyclic set; it is a $k$-majority tournament. We then compose copies of $T_{1}$ to obtain larger $k$-majority tournaments having no large acyclic sets.

For tournaments $T$ and $T^{\prime}$, the composition $T \circ T^{\prime}$ is the tournament obtained by replacing each vertex $u$ in $T$ with a copy $T^{\prime}(u)$ of $T^{\prime}$ and replacing each edge $u v$ in $T$ with an orientation of a complete bipartite graph with all edges directed from $T^{\prime}(u)$ to $T^{\prime}(v)$. Formally, if $V(T)=[r]$ and $V\left(T^{\prime}\right)=\left[r^{\prime}\right]$, then $V\left(T \circ T^{\prime}\right)=[r] \times\left[r^{\prime}\right]$, and $\left(x, x^{\prime}\right)\left(y, y^{\prime}\right)$ is an edge in $T \circ T^{\prime}$ if and only if (1) $x y \in E(T)$ or $(2) x=y$ and $x^{\prime} y^{\prime} \in E\left(T^{\prime}\right)$.

Proposition 3.2. If $T$ and $T^{\prime}$ are $k$-majority tournaments, then $T \circ T^{\prime}$ is a $k$-majority tournament.

Proof. Let $T$ and $T^{\prime}$ be $k$-majority tournaments on $[r]$ and $\left[r^{\prime}\right]$, respectively. Let $T$ be realized by $\left\{\pi_{1}, \ldots, \pi_{k}\right\}$ and $T^{\prime}$ be realized by $\left\{\sigma_{1}, \ldots, \sigma_{k}\right\}$. We construct a realizer $\left\{\tau_{1}, \ldots, \tau_{k}\right\}$ for $T \circ T^{\prime}$ by letting $\tau_{t}$ be the linear ordering of $[r] \times\left[r^{\prime}\right]$ obtained by replacing 
the occurrence of $i \in[r]$ in $\pi_{t}$ with $\left(i, \sigma_{t}(1)\right),\left(i, \sigma_{t}(2)\right), \ldots,\left(i, \sigma_{t}\left(r^{\prime}\right)\right)$, where $\sigma_{t}(j)$ is the $j$ th element of $\sigma_{t}$.

Consider an edge $\left(x, x^{\prime}\right)\left(y, y^{\prime}\right) \in E\left(T \circ T^{\prime}\right)$. If $x \neq y$, then $x y \in E(T)$, and hence more than half of $\pi_{1}, \ldots, \pi_{k}$ list $x$ before $y$. The corresponding orders in $\left\{\tau_{1}, \ldots, \tau_{k}\right\}$ list all elements with first coordinate $x$ before all elements with first coordinate $y$. If $x=y$, then $x^{\prime} y^{\prime} \in E\left(T^{\prime}\right)$, and hence more than half of $\sigma_{1}, \ldots, \sigma_{k}$ list $x^{\prime}$ before $y^{\prime}$. The corresponding orders in $\left\{\tau_{1}, \ldots, \tau_{k}\right\}$ list $\left(x, x^{\prime}\right)$ before $\left(y, y^{\prime}\right)$. It follows that $\tau_{1}, \ldots, \tau_{k}$ realize $T \circ T^{\prime}$.

Proposition 3.3. $a\left(T \circ T^{\prime}\right)=a(T) a\left(T^{\prime}\right)$.

Proof. If $S$ is acyclic in $T$ and $S^{\prime}$ is acyclic in $T^{\prime}$, then $S \times S^{\prime}$ is acyclic in $T \circ T^{\prime}$, so $a\left(T \circ T^{\prime}\right) \geq a(T) a\left(T^{\prime}\right)$. Conversely, if $\hat{S}$ is acyclic in $T \circ T^{\prime}$, then let $S=\{u \in$ $V(T):(u, v) \in \hat{S}$ for some $\left.v \in V\left(T^{\prime}\right)\right\}$. Note that $S$ is acyclic in $T$, since a cycle induced by $S$ lifts to a cycle induced by $S$. Also, for $u \in V(T)$, at most $a\left(T^{\prime}\right)$ vertices with first coordinate $u$ lie in $\hat{S}$. Thus $a\left(T \circ T^{\prime}\right) \leq|S| a\left(T^{\prime}\right) \leq a(T) a\left(T^{\prime}\right)$.

Proposition 3.4. Let $T_{1}$ be an n-vertex tournament, and let $\alpha=a\left(T_{1}\right)$. If $T_{j}=T_{j-1} \circ T_{1}$ for $j>1$, then $a\left(T_{j}\right)=\mid V\left(T_{j}\right)^{\mid \frac{\lg \alpha}{\lg n}}$.

Proof. Note that $\left|V\left(T_{j}\right)\right|=n^{j}$. Since $\alpha^{j \lg n}=n^{j \lg \alpha}$, Proposition 3.3 yields $a\left(T_{j}\right)=\alpha^{j}=$ $\mid V\left(T_{j}\right)^{\frac{\lg \alpha}{\lg n}}$.

Proposition 3.4 provides a way of building larger $k$-majority tournaments from an initial $k$-majority tournament $T_{1}$; when $a\left(T_{1}\right)$ is small, also $a\left(T_{j}\right)$ is small. A randomized construction produces a tournament with a given number of vertices that has no large acyclic set, but such tournaments typically are not $k$-majority tournaments. Nevertheless, when the given number of vertices is at most $(k-1) / 2$, every tournament is a $k$-majority tournament. Stronger results are known, but our result only needs the following simple proposition.

Proposition 3.5. Every n-vertex tournament is a $(2 n-1)$-majority tournament.

Proof. Let $T$ be an orientation of $K_{n}$. It is well known that $K_{n}$ is $n$-edge-colorable. Let $M_{1}, \ldots, M_{n}$ be a decomposition of $K_{n}$ into matchings. We first construct a realizer $\Pi$ of $T$ with $|\Pi|=2 n$. Each matching contributes two linear orders to $\Pi$. Let $M_{j}=\left\{u_{1} v_{1}, \ldots, u_{t} v_{t}\right\}$ with $u_{i} v_{i} \in E(T)$, and let $w_{1}, \ldots, w_{n-2 t}$ be the vertices not covered by $M_{j}$. The two orders generated by $M_{j}$ are $\left(u_{1}, v_{1}, \ldots, u_{t}, v_{t}, w_{1}, \ldots, w_{n-2 t}\right)$ and $\left(w_{n-2 t}, \ldots, w_{1}, u_{t}, v_{t}, \ldots, u_{1}, v_{1}\right)$.

All vertex pairs are neutral in the two orders except the edges of $M_{j}$ itself. Each edge of $T$ appears in one matching. Hence if $u v \in E(T)$, then $u$ appears before $v$ exactly $n+1$ times, so $\Pi$ realizes $T$. Furthermore, deleting any one member of $\Pi$ leaves $u$ before $v$ in at least $n$ of the remaining $2 n-1$ orders.

We now have the tools needed to prove our upper bound on $f_{k}(n)$ for general $k$.

Theorem 3.6. For $k$ fixed, $f_{k}(n) \leq n^{d_{k}(n)}$, where $d_{k}(n) \rightarrow \frac{1+\lg \lg k}{-1+\lg k}$ as $n \rightarrow \infty$. 
Proof. Let $k^{\prime}=(k+1) / 2$. By the result of Erdős and Moser [6], there is a $k^{\prime}$-vertex tournament $T_{1}$ with $a\left(T_{1}\right) \leq 1+2 \lg k^{\prime}$. Let $\alpha=a\left(T_{1}\right)$. By Proposition 3.5, $T_{1}$ is a $k$-majority tournament. Note also that $1+2 \lg k^{\prime} \leq 2 \lg k$ for $k \geq 3$, so $\alpha \leq 2 \lg k$.

Let $n$ be a positive integer, and let $n^{\prime}$ be the least power of $k^{\prime}$ that is at least as large as $n$. Note that $n^{\prime} \leq n k^{\prime}$. By Proposition 3.4, there is a $k$-majority tournament $T$ on $n^{\prime}$ vertices with $a(T)=\left(n^{\prime}\right)^{\frac{\lg \alpha}{\lg k^{\prime}}}$. Also $\lg k^{\prime}>-1+\lg k$. Hence

$$
f_{k}(n) \leq f_{k}\left(n^{\prime}\right) \leq\left(n^{\prime}\right)^{\frac{\lg \alpha}{\lg k^{\prime}}} \leq\left(n k^{\prime}\right)^{\frac{\lg \alpha}{\lg k^{\prime}}}=n^{\frac{\lg \alpha}{\lg k^{\prime}}\left(1+\frac{\lg k^{\prime}}{\lg n}\right)}<n^{\frac{1+\lg \lg k}{-1+\lg k}\left(1+\frac{\lg k}{\lg n}\right)} .
$$

As desired, the exponent tends to $\frac{1+\lg \lg k}{-1+\lg k}$ as $n \rightarrow \infty$.

Erdős and Moser [6] also proved that every $n$-vertex tournament is a $k$-majority tournament for $k=O(n / \log n)$; equivalently, for some constant $c$ every tournament on $c k \log k$ vertices is a $k$-majority tournament. Thus we could let $T_{1}$ be a tournament with $c k \log k$ vertices such that $a\left(T_{1}\right) \leq 3 \lg (c k \log k)$. This would produce a very slight improvement in our bound, increasing the denominator of the exponent by a lower order term.

\section{References}

[1] N. Alon, G. Brightwell, H.A. Kierstead, A.V. Kostochka, and P. Winkler, Dominating sets in k-majority tournaments, J. Combin. Theory Ser. B 96 (2006), 374-387.

[2] P. Beame, E. Blais, and D.T. Huynh-Ngoc, Longest common subsequences in sets of permutations, Arxiv preprint arXiv:0904.1615, 2009

[3] P. Beame, and D.T. Huynh-Ngoc, On the value of multiple read/write streams for approximating frequency moments, IEEE 49th Annual IEEE Symposium on Foundations of Computer Science, 2008. FOCS'08, (2008), 499-508

[4] R. P. Dilworth, A decomposition theorem for partially ordered sets. Ann. of Math. (2) 51 (1950), 161-166.

[5] P. Erdős, On a problem in graph theory, Math. Gaz. 47 (1963), 220-223.

[6] P. Erdős and L. Moser, On the representation of directed graphs as unions of orderings, Magyar Tud. Akad. Mat. Kutató Int. Közl. 9 (1964), 125-132.

[7] P. Erdős, and G. Szekeres, A combinatorial problem in geometry, Compositio Math. 2 (1935), 463-470.

[8] D.C. McGarvey, A theorem on the construction of voting paradoxes, Econometrica 21 (1953), 608-610.

[9] R. Stearns, The voting problem, Amer. Math. Monthly 66 (1959), 761-763. 